\title{
Mise en place d'une plate-forme d'essais pour l'étude dynamique d'amortisseurs automobile
}

\author{
Philippe Viota et Christophe Lemaire \\ LAMEFIP, ENSAM de Bordeaux, 33405 Talence Cedex, France
}

Reçu le 27 octobre 2005

\begin{abstract}
Résumé - Dans le secteur automobile, depuis plusieurs années, les amortisseurs utilisés dans les suspensions font l'objet de nombreuses études. Afin d'améliorer leurs performances, leurs comportements doivent être connus et cette identification nécessite des essais appropriés. Un vérin grande vitesse a été mis en oeuvre au laboratoire LAMEFIP afin de caractériser des structures et des matériaux sous impacts. Parce que le pilotage de cet actionneur hydraulique permet de piloter le déplacement de la tige du vérin et d'imposer par exemple de grande vitesse de déplacement constante pendant le choc (vitesse maximum $5 \mathrm{~m} / \mathrm{s}$ ), ce nouveau dispositif a été utilisé pour la première fois pour caractériser en dynamique un amortisseur utilisé habituellement dans une suspension de véhicule tout terrain. Les premiers essais réalisés avec ce vérin dynamique ont permis d'estimer les performances mécaniques de ce nouveau dispositif expérimental de sollicitations dynamiques et d'identifier en sus le comportement mécanique de cet amortisseur.
\end{abstract}

Mots clés : Vérin grande vitesse / impact / crash / sollicitation dynamique / amortisseur automobile

Abstract - In automobile sector, for several years, dampers used in suspensions have made the object of an important research. In order to improve their performances, the identification of their behaviour has to be known and can be achieve from appropriate tests. A dynamic jack was developed at LAMEFIP laboratory to characterize structures and materials under impacts. Because the control system of this hydraulic actuator makes it possible to pilot the jack rod displacement and to impose for example a high constant speed of displacement during the shock - maximum speed $5 \mathrm{~m} / \mathrm{s}-$, this new apparatus was used for the first time in order to dynamically characterize a shock absorber installed usually into all road vehicle suspensions. The mechanical behaviour of the car shock absorber was characterized from these impact tests.

Key words: High speed jack / impact / crash / dynamical loading / automotive damper

\section{Introduction}

Depuis plusieurs années, les amortisseurs de suspension automobile sont largement étudiés afin d'améliorer la tenue de route des véhicules. Ces amortisseurs font en effet partie des pièces maîtresses qui assurent le bon comportement de la voiture. L'identification du comportement de l'amortisseur - qu'il soit de type classique (comportement passif) ou s'intégrant dans une suspension semi-active - a plusieurs objectifs. Cette identification

a Auteur correspondant :

P. Viot, philippe.viot@lamef.bordeaux.ensam.fr permet par exemple une optimisation des performances du composant - en compétition automobile, un réglage fin de l'amortisseur peut être effectué en fonction du circuit de la course pour améliorer la tenue de route de la voiture - . En recherche et développement, à partir d'une identification expérimentale de la réponse de l'amortisseur, un modèle de comportement peut être proposé et implémenté dans des codes de calcul.

Une demi suspension avant de véhicule peut être modélisée comme le présente la figure 1 (voir Gobbi \& Mastinu (2001) [1], Naudé \& Snyman (2003) [2,3]). La roue, le moyeu et le bras de suspension constituent un ensemble mécanique de masse $m$. La rigidité du pneu peut 


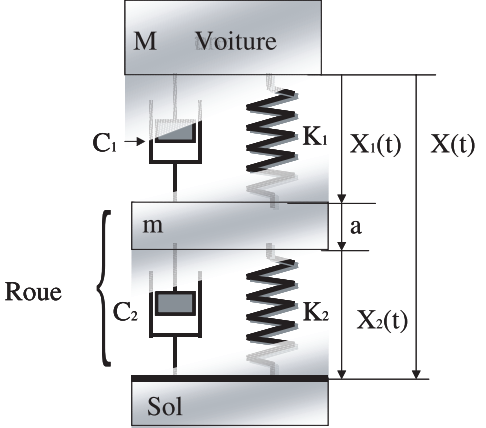

Fig. 1. Schéma d'une suspension automobile.

être introduite par l'intermédiaire d'un ressort - de rigidité $K_{2}$ - et d'un amortisseur $C_{2}$ montés en parallèle. Ces pièces mobiles sont en liaison avec la structure (masse $M$ ) supposée rigide de la voiture via plusieurs pièces (barres stabilisatrices, ... ) qui dépendent évidemment de la morphologie de la suspension. Cependant, quelle que soit son architecture, un ressort de rigidité $K_{1}$ et un amortisseur de coefficient $C_{1}$ peuvent modéliser les organes de la suspension qui transmettent les forces entre le véhicule et la route.

La simulation numérique de la suspension - pour étudier sa réponse à une sollicitation dynamique - requiert nécessairement l'identification de ces paramètres $K_{1}, K_{2}, C_{2}$ et pour ce qui nous intéresse plus particulièrement la caractérisation fine de la réponse $C_{1}$ de l'amortisseur automobile.

Cette identification peut être obtenue par des expériences appropriées. In situ, par une instrumentation de la voiture (dispositif expérimental embarqué), il est possible de mesurer directement le comportement global de la suspension, sa cinématique et les efforts qui transitent à travers les pièces. Ces expériences sont en général difficiles à mettre en œuvre, coûteuses et, de ce fait, elles sont utilisées pour valider des solutions technologiques sur des prototypes déjà bien aboutis. Il est donc nécessaire de développer aussi, en laboratoire, des dispositifs expérimentaux qui peuvent imposer des chargements dynamiques ou des déplacements contrôlés sur un amortisseur seul (voir Fischer \& Iserman [4]).

Le laboratoire LAMEFIP s'est récemment doté d'un vérin grande vitesse dans l'objectif de tester des matériaux et des structures sous chargements dynamiques. Le développement et la mise en oeuvre de ce nouveau dispositif expérimental a été défini, à partir d'un cahier des charges précis (qui indiquait entres autres les caractéristiques mécaniques du vérin et les performances du système de pilotage), en collaboration avec la société TEMA CONCEPT chargée de la fabrication. Sans détailler l'ensemble des spécificités du dispositif, on peut retenir que les capacités maximales en force varient de $100 \mathrm{KN}$ pour les faibles vitesses à $10 \mathrm{KN}$ pour les essais d'impact (à la vitesse maximum de $5 \mathrm{~m} / \mathrm{s}$ ). L'originalité de ce prototype de machine d'essai réside principalement dans le système de pilotage qui doit être capable de contrôler la vitesse de déplacement de la tige du vérin et ceci quelle que soit la réponse de la structure testée (dans la gamme des efforts de la machine). De nombreuses machines d'essai de sollicitations rapides se comportent en fait comme des catapultes; une vitesse initiale est programmée et la vitesse d'impact évolue en fonction du comportement de la structure testée. Il est donc dans ce cas assez difficile d'identifier la réponse d'un matériau ou d'une structure à une vitesse de déformation donnée. L'ambition de ce projet réside principalement dans la définition d'un vérin hydraulique capable d'atteindre des vitesses élevées de sollicitations contrôlées par un système de pilotage robuste (une thèse actuellement en cours au laboratoire a pour objectif l'optimisation de l'asservissement de l'actionneur).

Pour estimer les performances de ce dispositif original de sollicitation dynamique, il était nécessaire de tester une structure mécanique capable de supporter des déplacements de grandes amplitudes. Ce vérin semble ainsi particulièrement approprié pour caractériser le comportement dynamique d'un amortisseur automobile. Il est en effet possible d'imposer des déplacements supérieurs à $100 \mathrm{~mm}$, à des vitesses élevées proches de celles imposées sur l'amortisseur en utilisation - de l'ordre de 2 à $3 \mathrm{~m} / \mathrm{s}$ - et sous des efforts significatifs. Il a donc été naturellement envisagé d'effectuer des essais sur des amortisseurs automobiles. De ce fait, cette étude a un double objectif : premièrement, valider les performances du vérin grande vitesse et deuxièmement identifier le comportement d'un amortisseur utilisé dans les véhicules tout terrain.

\section{Dispositif expérimental}

Le vérin est positionné verticalement et fixé sur une dalle sismique (Fig. 2). L'échantillon (ou la structure à tester) est fixé au-dessus de la tête d'impact du piston sur une structure modulaire constituée de poutrelles en IPN et de deux plateaux rigides. Le vérin est un actionneur hydraulique double effet. La tige du vérin est guidée par deux paliers hydrostatiques et son déplacement maximal est de $600 \mathrm{~mm}$. Elle est équipée de deux butées hydrauliques de fin de course - d'une longueur de $30 \mathrm{~mm}$ - pour éviter tout choc entre le piston et le corps du vérin. La pression hydraulique étant de 280 bars, la force maximale est de $106 \mathrm{KN}$ en quasi statique. À la vitesse maximum de $5 \mathrm{~m} / \mathrm{s}$, les capacités du dispositif hydraulique limitent la force axiale à $10 \mathrm{KN}$.

Le groupe hydraulique Schenk comprend une pompe entraînée par un moteur électrique, un réservoir, des filtres et des vannes de sécurité. Cette unité hydraulique est installée dans un local insonorisé et la puissance hydraulique est transmise via des flexibles à 3 accumulateurs haute pression (de 10 litres chacun). Ces accumulateurs assurent une réserve d'huile de 280 bars utilisée pour alimenter directement les deux servovalves Hydrostar (de 3 étages et de 600 litres montées en parallèle). Trois accumulateurs complémentaires sont utilisés pour récupérer l'huile provenant du vérin pendant un tir.

Les deux servovalves (commandées par le poste de pilotage) contrôlent les pressions dans les chambres (haute 


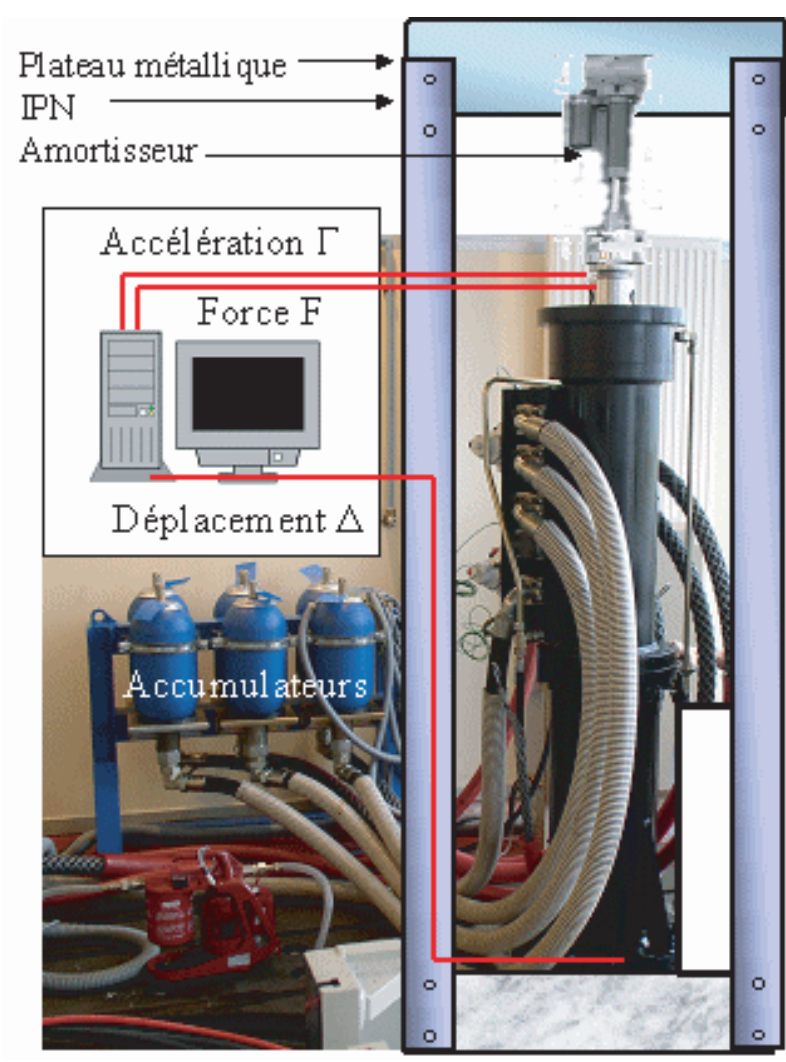

Fig. 2. Le vérin et ses équipements.

et basse) du vérin et imposent ainsi le déplacement de la tige.

En ce qui concerne l'instrumentation du dispositif, on retrouve les capteurs classiques nécessaires au pilotage du vérin (capteurs de pression dans les chambres supérieures et inférieures, capteurs de position des servovalves ...) et plusieurs capteurs utilisés pour mesurer les caractéristiques de l'essai. Un capteur piézoélectrique (force maximum $\mathrm{F}$ de $100 \mathrm{KN}$ ) et un accéléromètre (capacité $\Gamma=100 \mathrm{~g}$ ) sont placés sur la tête du piston, au plus près du point d'impact. L'accéléromètre est placé à l'intérieur de la tête du piston pour qu'il ne subisse pas d'effort mécanique. Un capteur magnéto-strictif de déplacement est fixé dans la partie basse du vérin. Cette instrumentation de base peut être complétée par des mesures extensométriques ou des films obtenus avec une caméra rapide Phantom V4 dont la résolution est de $512 \times 512$ pixels $^{2}$ à une fréquence de 2000 images par seconde. Au total, 16 voies analogiques simultanées peuvent être utilisées pour enregistrer les caractéristiques de l'essai et la réponse de la structure.

Le pilotage du vérin permet la définition des cycles classiques d'une machine d'essai hydraulique : sinus, cycles en triangle, demi-sinus et rampe. Le dispositif de commande permet aussi de générer une courbe quelconque de déplacement en fonction du temps à partir de données enregistrées dans un fichier. Cette option est particulièrement intéressante pour simuler par exemple le choc réel d'une roue sur un trottoir, le passage d'une bosse ...

Les premiers essais ont été réalisés 《à vide » sans aucune structure à impacter. Dans cette configuration, les capacités dynamiques du dispositif ont été confirmées pour chaque cycle. Le logiciel de commande pilote précisément le déplacement de la tige. Cette première vérification ne permet pas cependant de valider les performances de l'actionneur sous charge.

L'utilisation d'un amortisseur automobile comme structure d'essai permet la réalisation d'impacts avec des grands déplacements et des efforts significatifs. De plus, pendant un tir (sans défaillances de pilotage), «l'échantillon»n'est pas détruit et peut subir une campagne complète d'expériences. Ainsi, il est possible de vérifier les performances du dispositif complet de tir. Avec ces mêmes résultats, on peut bien entendu estimer la réponse dynamique de l'amortisseur et évaluer l'influence des réglages.

\section{Résultats}

\subsection{Validation des performances du vérin}

Les premiers essais - cycle en sinus - ont été effectués sur un amortisseur de véhicule tout terrain utilisé en compétition automobile. Il a été possible d'identifier les caractéristiques du dispositif hydraulique complet, et en particulier, de valider ses performances en terme de force et de vitesse. Des cycles en sinus ont été choisis pour augmenter progressivement vitesse et accélération et réduire les risques de dommage sur le composant et sur le vérin. Le déplacement imposé $d_{c}$ de ces essais est défini classiquement par l'équation :

$$
d_{c}=\frac{d_{\max }}{2} \cdot \sin (2 . \pi . f . t)
$$

L'amplitude du déplacement $d_{\max }$ (crête à crête) limité à $84 \mathrm{~mm}$ est imposée par les caractéristiques géométriques de l'amortisseur. La fréquence $f$ des cycles est augmentée progressivement pour atteindre $16 \mathrm{~Hz}$. Des essais complémentaires ont été effectués avec des amplitudes de déplacement plus faibles et des fréquences plus élevées. Cependant, les trois accumulateurs haute pression, limités en volume d'huile, n'autorisent pas des cycles grand déplacement - haute fréquence.

On peut comparer (Fig. 3) l'évolution des déplacements réels et imposés pour les trois cycles. Un écart temporel constant $\delta t$ est observé - inférieur à $20 \mathrm{~ms}$ - qui correspond au temps de réaction de l'actionneur.

Sur cette même figure, on montre que les amplitudes des déplacements ne peuvent être atteintes pour les fréquences les plus élevées. À la fréquence de $16 \mathrm{~Hz}$, par exemple, le déplacement effectif du piston est $38 \%$ inférieur à la commande. En fait, avec l'amortisseur fixé sur la tige du vérin, plus la fréquence est élevée, plus la force engendrée par l'amortisseur augmente et dans cette 


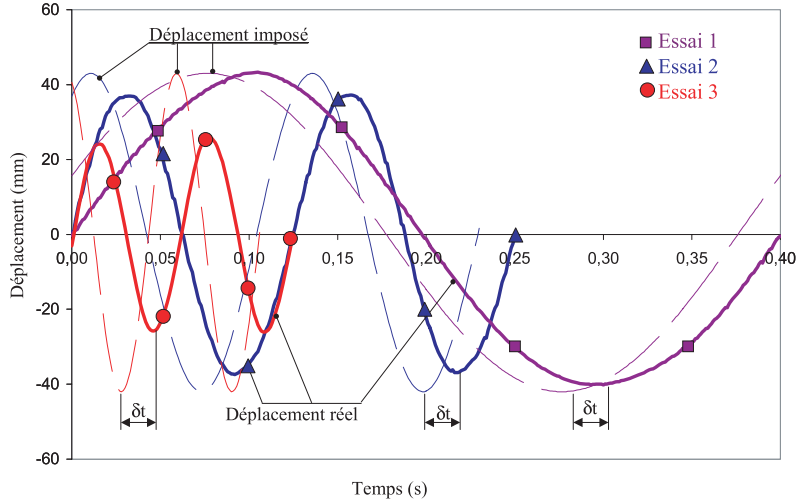

Fig. 3. Déplacement imposé (ligne fine) et réel (ligne épaisse) en fonction du temps.

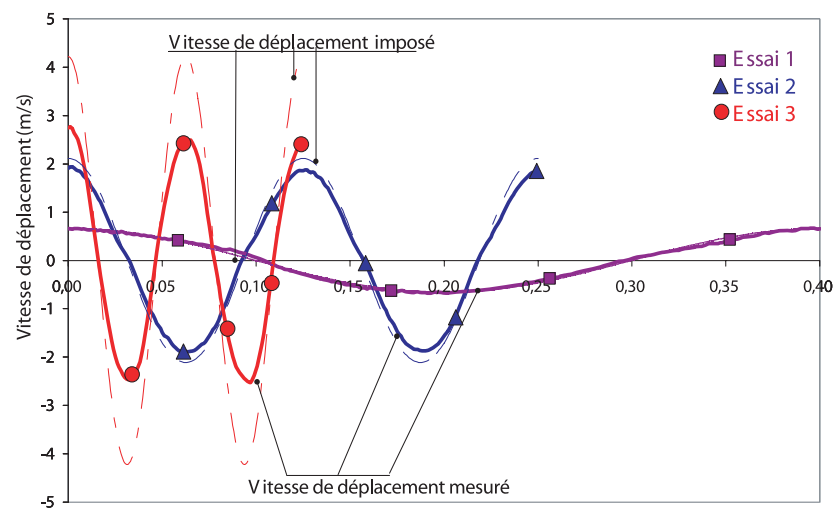

Temps (s)

Fig. 4. Évolution de la vitesse de déplacement en fonction du temps pour les trois essais.

Tableau 1. Caractéristiques des cycles sinus.

\begin{tabular}{cccccc}
\hline & \multicolumn{3}{c}{ Caractéristiques d'essai } & \multicolumn{2}{c}{ Résultats } \\
\hline essai & $\begin{array}{c}\text { Fréq. } \\
(\mathrm{Hz})\end{array}$ & $\begin{array}{c}d_{\max } \\
(\mathrm{mm})\end{array}$ & $\begin{array}{c}V_{\max } \\
(\mathrm{m} / \mathrm{s})\end{array}$ & $\begin{array}{c}d_{r . \max } \\
(\mathrm{mm})\end{array}$ & $\begin{array}{c}V_{r . \max } \\
(\mathrm{m} / \mathrm{s})\end{array}$ \\
\hline 1 & 2,5 & \pm 42 & 0,66 & \pm 42 & 0,66 \\
2 & 8 & \pm 42 & 2,10 & \pm 37 & 1,86 \\
3 & 16 & \pm 42 & 4,22 & \pm 26 & 2,61 \\
\hline
\end{tabular}

configuration, les limites dynamiques du vérin sont atteintes.

En conséquence, les vitesses mesurées pendant ces cycles ne correspondent pas aux vitesses imposées (Fig. 4). À l'exception des faibles fréquences (à $4 \mathrm{~Hz}$, essai 1 ), les valeurs maximum des vitesses effectives $v_{r \text { max }}$ sont plus faibles que les vitesses théoriques $v_{\max }$ (Tab. 1). On trouve évidemment les mêmes écarts temporels sur les vitesses que sur les déplacements puisque les fréquences mesurées sont égales aux consignes. En conclusion, la dérivée de l'équation (1) ne peut donner l'évolution réelle de la vitesse, il faut prendre en compte l'amplitude réelle du déplacement $d_{r \text { max }}$ pour retrouver l'évolution temporelle de la vitesse. On obtient alors :

$$
v_{c}=d_{r \cdot \max } \cdot \pi \cdot f \cdot \cos (2 \cdot \pi \cdot f \cdot t)
$$

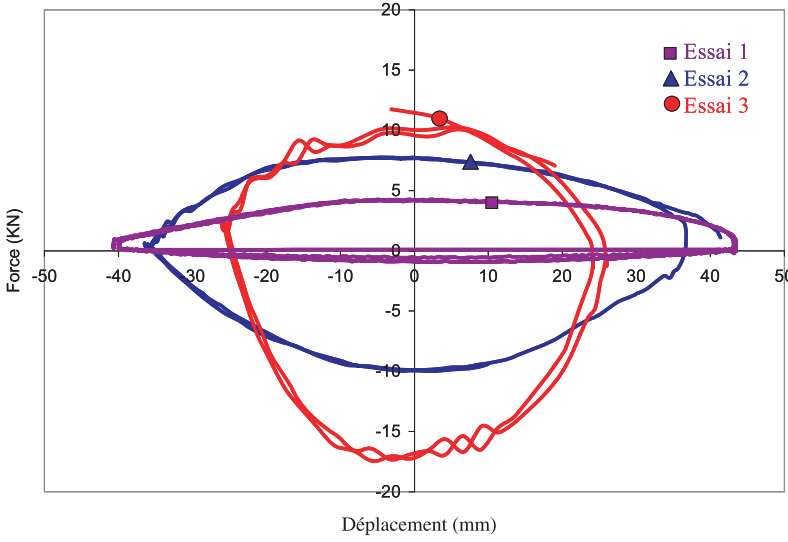

Fig. 5. Évolution de la force en fonction de la vitesse.

Ces essais permettent aussi d'identifier la réponse de l'amortisseur à une sollicitation cyclique. Il faut souligner que ces résultats sont obtenus avec des réglages d'amortisseur déterminés en usine par le constructeur. Habituellement, les amortisseurs automobiles de compétition peuvent être réglés afin de modifier indépendamment la réponse du composant pour les deux modes de chargement - compression et détente.

La figure 5 présente la réponse de l'amortisseur pour les trois cycles. Par convention, la force est positive quand l'amortisseur est comprimé, et devient négative (détente) dans la direction opposée. Le comportement de l'amortisseur est asymétrique, il dépend du sens de chargement (compression ou détente). Cependant, quelle que soit la direction de sollicitation, une augmentation du niveau des efforts peut être constatée en relation avec l'augmentation de la vitesse de déplacement. Pour le test 3 (à $16 \mathrm{~Hz}$ ), la force de détente atteint même $18 \mathrm{KN}$.

En conclusion, les performances dynamiques du vérin sous chargement ont été évaluées à partir de ces premiers essais appliqués sur un amortisseur automobile. Les limites du dispositif expérimental ont pu être détectées : pour l'essai 3, l'amortisseur applique un effort élevé de $18 \mathrm{KN}$ et la vitesse du vérin est de $2,6 \mathrm{~m} / \mathrm{s}$. Ce couple de paramètres semble être un point de fonctionnement du vérin.

Pour compléter cette étude et décrire plus précisément l'enveloppe des points force- vitesse de ce nouveau dispositif, des expériences complémentaires ont été menées sur d'autres structures. Il a été réalisé des compressions sur des échantillons de nid d'abeille et d'élastomères de différentes sections, et des tubes d'aluminium (compression axiale faisant apparaître du flambement) en imposant des vitesses d'impact variables. Les capacités en effort du vérin en fonction de la vitesse ont donc pu être un peu mieux identifiées (Fig. 6). Pour les vitesses supérieures à $2 \mathrm{~m} / \mathrm{s}$, on constate des écarts sensibles entre les résultats suivant les configurations d'essais et les structures sollicitées. Ceci montre clairement un manque de robustesse dans le système de pilotage du vérin. L'étude en cours doit permettre l'amélioration de l'asservissement de l'ensemble. 


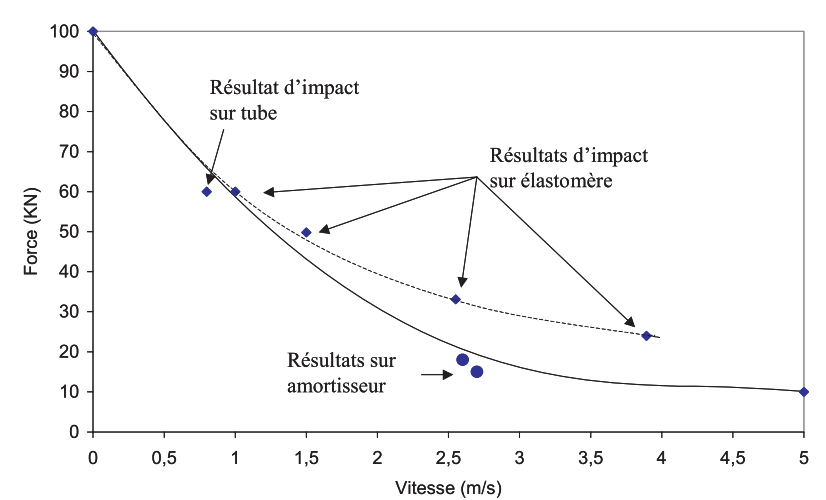

Fig. 6. Évolution du déplacement en fonction du temps.

Tableau 2. Caractéristiques des essais d'impact.

\begin{tabular}{ccccc}
\hline \multicolumn{3}{c}{ Compression } & Détente \\
\hline Essai & $\begin{array}{c}V_{c} \\
(\mathrm{~m} / \mathrm{s})\end{array}$ & $\begin{array}{c}\text { Déplacement } \\
(\mathrm{mm})\end{array}$ & $\begin{array}{c}V_{d} \\
(\mathrm{~m} / \mathrm{s})\end{array}$ & $\begin{array}{c}\text { Déplacement } \\
(\mathrm{mm})\end{array}$ \\
\hline 1 & 0,3 & & 0,3 & \\
2 & 1 & 85 & 0,5 & 0 \\
3 & 3 & & 0,7 & 0 \\
\hline 3bis & 0,3 & 96 & 0,3 & \\
\hline
\end{tabular}

Concernant l'amortisseur automobile testé, sa réponse dynamique a été évaluée et ses limites en effort sont maintenant connues. Des essais d'impact ont donc pu être réalisés afin de compléter l'identification de cet amortisseur.

\subsection{Essais d'impact}

Les cycles imposés pour ces tests comprennent trois étapes : premièrement, une montée de la tige du vérin de $86 \mathrm{~mm}$ (phase de compression de l'amortisseur) à une vitesse stabilisée $V_{c}$, suivie par un palier de 0,2 seconde. L'essai se termine par une détente de l'amortisseur à la vitesse $V_{d}$ pour revenir à la position initiale. Les vitesses de compression et de détente ont été choisies en fonction des capacités de l'amortisseur (Tab. 2). Il est nécessaire que les efforts supportés par l'amortisseur ne dépasse pas une valeur seuil $F_{m}$ spécifiée par le fabricant. Les vitesses de détente ont été volontairement choisies plus faibles pour ne pas endommager l'amortisseur. En utilisation classique, ces vitesses dépassent rarement $1 \mathrm{~m} / \mathrm{s}$. En revanche, en compression, il est possible d'atteindre $3 \mathrm{~m} / \mathrm{s}$. Un essai supplémentaire a été réalisé en imposant un déplacement de $96 \mathrm{~mm}$ pour évaluer l'effet de la butée hydraulique de l'amortisseur.

La figure 7 présente les cycles imposés et les déplacements réels effectués par la tige du vérin. On peut observer pendant les phases de compression et de détente un écart temporel de $20 \mathrm{~ms}$ entre la consigne et le déplacement effectif. Cependant, cet écart temporel est sans conséquence sur les résultats attendus concernant l'amortisseur. À la fin de cette première étape, la vitesse de la tige chute évidemment pour que la tige du vérin atteigne la consigne en déplacement avec une précision

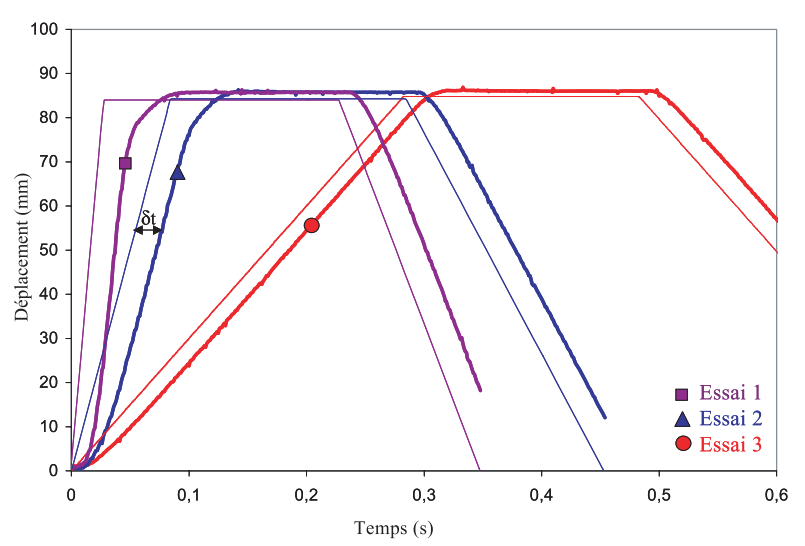

Fig. 7. Évolution du déplacement en fonction du temps.

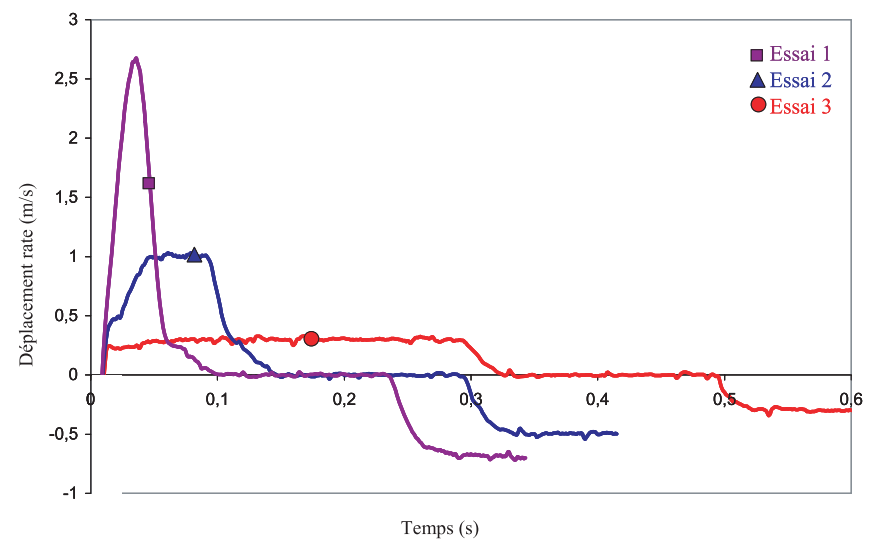

Fig. 8. Évolution de la vitesse de déplacement en fonction du temps.

de $1 \mathrm{~mm}$. Les réglages PID du système de pilotage ont été définis pour que le vérin atteigne le plus rapidement la consigne en vitesse sans pour autant qu'apparaisse un dépassement significatif de la consigne en déplacement.

Pendant les rampes de déplacement, les vitesses sont imposées constantes. Pour les expériences (1) et (2), les vitesses atteintes correspondent bien aux valeurs imposées (Fig. 8). On obtient, par exemple pour l'essai 2, une vitesse de compression de $1 \mathrm{~m} / \mathrm{s}$ pendant $80 \mathrm{~ms}$. En revanche, pendant cette même phase de compression pour l'essai 3, l'actionneur ne peut atteindre la vitesse consigne de $3 \mathrm{~m} / \mathrm{s}$; la valeur maximale est seulement de $2,7 \mathrm{~m} / \mathrm{s}$ - l'effort de réaction de l'amortisseur est 15 KN. Dans cette configuration d'essai, le couple (force $15 \mathrm{KN}$, vitesse $2.7 \mathrm{~m} / \mathrm{s}$ ) est un point de fonctionnement du vérin (Fig. 6).

Ces essais ont permis d'identifier le comportement de l'amortisseur sous impact. Les niveaux des efforts (Fig. 9) dépendent de la vitesse réelle imposée sur l'amortisseur. On montre que la réponse en force est une fonction de la vitesse et du sens de sollicitation (et des réglages de l'amortisseur). En fait, pour des vitesses de compression et de détente identiques, les forces engendrées lors de la détente sont nettement supérieures (essai 1). Pour les autres essais, l'influence de la vitesse de déplacement est aussi mise en évidence : on observe une augmentation de 


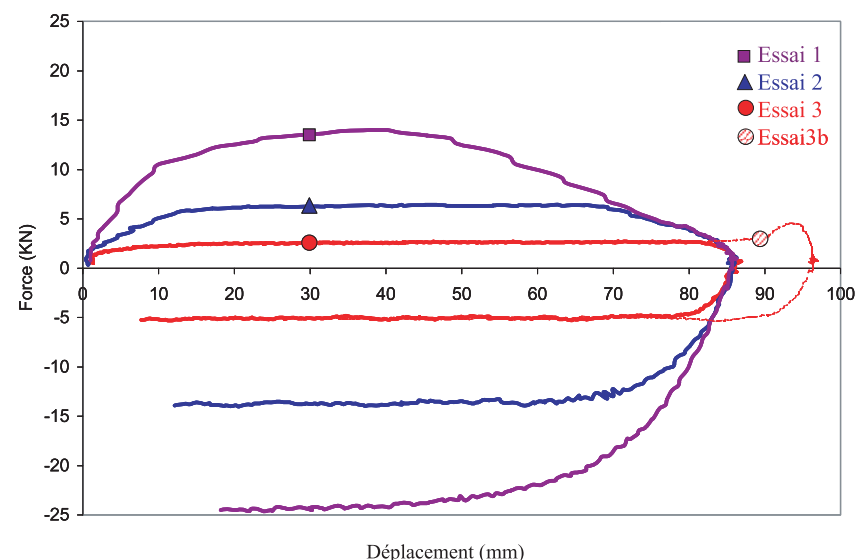

Fig. 9. Évolution de la force en fonction du déplacement.

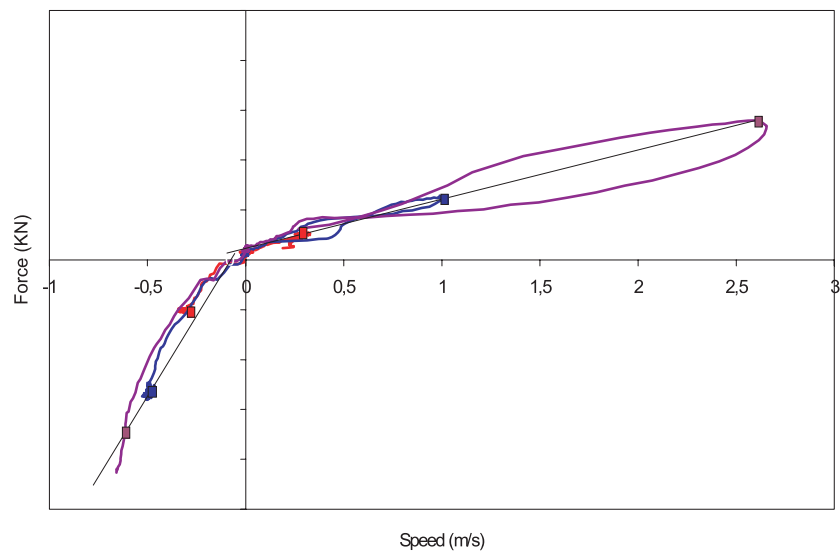

Fig. 10. Évolution de la force en fonction de la vitesse.

l'effort lorsque la vitesse augmente. De plus, les efforts en détente sont supérieurs à ceux mesurés en compression.

Pour l'essai 3bis, les conditions en vitesse sont identiques à celles de l'essai 1 et la réponse de l'amortisseur est équivalente. On a donc une bonne reproductibilité des résultats. En fin de compression de cet essai, la tige de l'amortisseur pénètre dans la butée hydraulique de $8 \mathrm{~mm}$; la force de compression augmente progressivement pour freiner le déplacement de la tige. La butée de fin de course de l'amortisseur semble donc efficace.

À partir de ces essais, les points caractéristiques force - vitesse du comportement de l'amortisseur ont été identifiés. Pour cette sollicitation compression/détente, l'évolution de la force n'est pas une simple fonction linéaire de la vitesse (dans la gamme des vitesses mesurées et pour ce composant particulier). Il est donc impossible d'établir un coefficient $C_{1}$ unique et constant. L'influence du sens du déplacement - compression ou détente - sur la réponse de l'amortisseur est en effet bien mis en évidence. Dans une première approche, le comportement global de l'amortisseur peut être éventuellement caractérisé par deux coefficients d'amortissement $C_{1}$ (en compression) et $C_{1}^{\prime}$ pour la détente comme le propose Fischer \& Isermann [4].
Cependant, l'évolution bi-linéaire de la force en fonction de la vitesse, et donc l'existence de deux coefficients d'amortissement constants, ne permet pas de représenter complètement la réponse du composant. Une modélisation plus fine doit être menée pour prendre en compte la non linéarité du comportement dans les phases de compression et de détente. Des phénomènes physiques (compressibilité de l'huile, phénomènes de cavitation, frottement entre pièces mécaniques ...) constatés pendant le fonctionnement de l'amortisseur doivent être modéliser pour proposer un modèle phénoménologique plus proche de la réalité et capable de mieux représenter les non linéarités et l'hystérésis du système.

\section{Conclusion}

Le vérin grande vitesse a été mis au point au laboratoire LAMEFIP pour effectuer des essais de sollicitation dynamique et d'impact à vitesse contrôlée. Les premières expériences ont montré que la vitesse maximum atteinte par l'actionneur hydraulique est de $5 \mathrm{~m} / \mathrm{s}$ et conforme aux spécifications du cahier des charges. Les essais complémentaires effectués sur un amortisseur de véhicule tout terrain (et sur d'autres structures) ont permis de caractériser les performances du vérin : ces expériences montrent que le dispositif complet (actionneur, ensemble hydraulique et unité de pilotage) est opérationnel pour caractériser les matériaux ou les structures sous chargement dynamique mais que le pilotage de l'ensemble doit être encore amélioré pour augmenter les capacités dynamiques de la machine.

Pendant cette étude, l'identification du comportement d'un amortisseur automobile a pu aussi être effectuée. Les essais d'impact ont montré l'influence de la vitesse sur la force de réaction de l'amortisseur. Ces résultats ont permis de déterminer les coefficients d'amortissement de l'amortisseur en compression et en traction.

\section{Références}

[1] M. Gobbi, G. Mastinu, Analytical description and optimisation of the dynamic behaviour of passively suspended road vehicles, J. of Sound and Vibration 245 (2001) 457-481

[2] A.F. Naudé, J.A. Snyman, Optimisation of road vehicle passive suspensions systems, Part 1. Optimisation algorithm and vehicle model, Applied Mathematical Modelling 27 (2003) 249-261

[3] A.F. Naudé, J.A. Snyman, Optimisation of road vehicle passive suspensions systems, Part 2. Qualification and case study, Applied Mathematical Modelling 27 (2003) 263-274

[4] D. Fischer, R. Isermann, Mechatronic semi-active and active vehicle suspensions, Control Engineering Practice 